\title{
Photon-Detection Timing-Jitter Model in Verilog-A
}

\author{
Juan Manuel López-Martínez, Ricardo Carmona-Galán and Ángel Rodríguez-Vázquez \\ Instituto de Microelectrónica de Sevilla-CNM \\ CSIC-Universidad de Sevilla \\ Sevilla, Spain \\ jmlopez@imse-cnm.csic.es
}

\begin{abstract}
Single-photon avalanche diodes can be employed to register the arrival of an individual photon. They are biased beyond breakdown voltage, and thus the electron-hole pairs generated by any incident photon is accelerated by the strong electric field triggering an avalanche current. In recent years, there have been attempts to model its characteristics in Verilog-A HDL. However, none of them have modelled its photon-detection timing jitter. This paper explains the mechanism of avalanche triggering and proposes a first approach to model it in Verilog-A. Comparison with experimental data and data reported in literature validates the model.
\end{abstract}

Keywords-Photon detection; timing jitter; Verilog-A; singlephoton avalanche diode (SPAD); device simulation.

\section{INTRODUCTION}

Single-photon avalanche diodes (SPADs) are semiconductor devices with the ability of detecting the arrival of isolated photons. They have been initially developed as a cheaper, magnetic-field-compatible replacement for photomultiplier tubes. They offer higher photon detection efficiency, especially in the red and near infrared spectrum. SPADs can be employed either to sense low-intensity luminous signals, by photon counting, or to timestamp the arrival these photons, in order to estimate their time-of-flight (ToF). Nowadays they can be built in CMOS technology, what allows incorporating functionality and opens the door to many more applications. Apart from depth and proximity sensors, SPADs are finding suitable application scenarios in fluorescence life-time microscopy (FLIM) [1] and fluorescence correlation spectroscopy [2].

SPADs are avalanche diodes operated in Geiger mode, that is, they are reverse biased above its breakdown voltage. In these conditions, anything can trigger a self-sustained avalanche like, for instance, a photon absorbed or a carrier generation event in depletion region. Over the last years there has been an interest to develop an accurate model of this device. However, its electrical characteristics and noise behavior have proven to be difficult to emulate. The most prominent models in the literature for these devices have been written in Verilog-A HDL, as it is fully compatible with a wide range of circuit simulators, like Cadence Spectre or Synopsys HSPICE. These models have been refined over time. For example, the first model of a SPAD using Verilog-A introduced the voltage dependence of the junction capacitance [3]. This model, however, lacked some important statistical phenomenon, like dark count noise (DCR) and afterpulsing probability (AP). Later on, DCR and AP were included [4][5]. However, band-to-band tunneling (BTBT), and the fact that AP has a strong dependence on the time since the previous avalanche event, was not considered in [3]. A more recent work, included trap-assisted tunneling and self-heating [7].

Despite all these advances, none of these models tried to include the SPAD photon-detection timing jitter. This is due to the architecture of the analytical model itself (Fig.1). It results in an almost instantaneous response time, as it depends on the time constants associated with the junctions and stray capacitances, which are in the order of femto-farads, and do not take into account the statistical behavior of the avalanche build up and the avalanche spreading dynamics throughout the active area. Also, the study of the photon-detection timing jitter of a SPAD requires the study of the device absorption coefficients, and the electric field profile on the device when it is operated in Geiger mode, which it is not easy to derive from the available information.

In this work, a photon-detection timing-jitter model was incorporated into an existing Verilog-A SPAD model [6], based in TCAD simulation of carrier dispersion and junction electric field profile in the SPAD.

\section{Simulated DeVICE}

The basic device structure is that of the work in [6]. It consists in a $12 \mu \mathrm{m}$-diameter active area diode with a guard ring of lowdoped $\mathrm{p}$-well material, or T-Well, around the central $\mathrm{p}+/$ deepn-well breakdown region, to avoid edge-junction breakdown (Fig.2). This SPAD was found to have a homogeneous breakdown voltage of $10.35 \mathrm{~V}$ throughout its active area at a temperature of $295 \mathrm{~K}$, with a maximum electric field of 0.729 $\mathrm{MV} / \mathrm{cm}$. It reached experimentally $187 \mathrm{ps}$ of timing jitter under an excess bias voltage of $0.9 \mathrm{~V}$.

\section{Photon-Detection Timing-Jitter Mechanics}

When a photon successfully strikes the active area region of SPAD, it can potentially create an electron-hole pair along its way inside the device. The probability of this photon to be detected by the SPAD is governed by the photon detection efficiency (PDE):

$$
\mathrm{PDE}=\mathrm{QE}(\lambda) \cdot \mathrm{FF} \cdot P_{t r}
$$

being $\mathrm{QE}(\lambda)$ the quantum efficiency, which can be determined by TCAD simulations, FF the fill factor and $P_{t r}$ the avalanche triggering probability. 


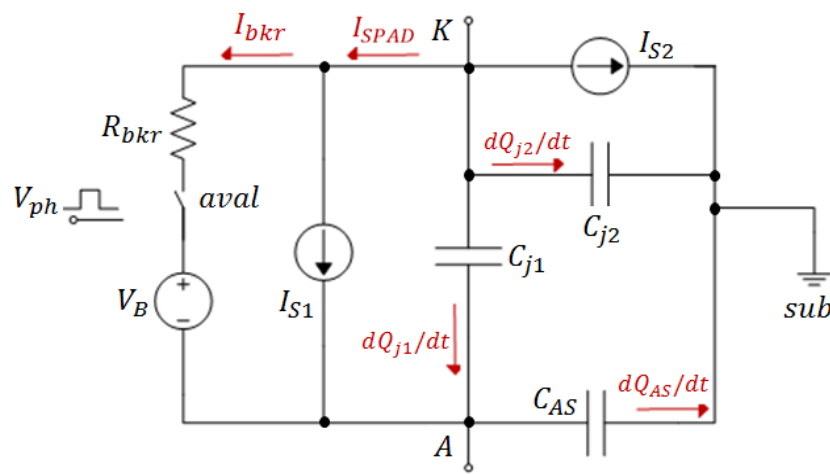

Fig. 1. SPAD analytical model proposed in [6].

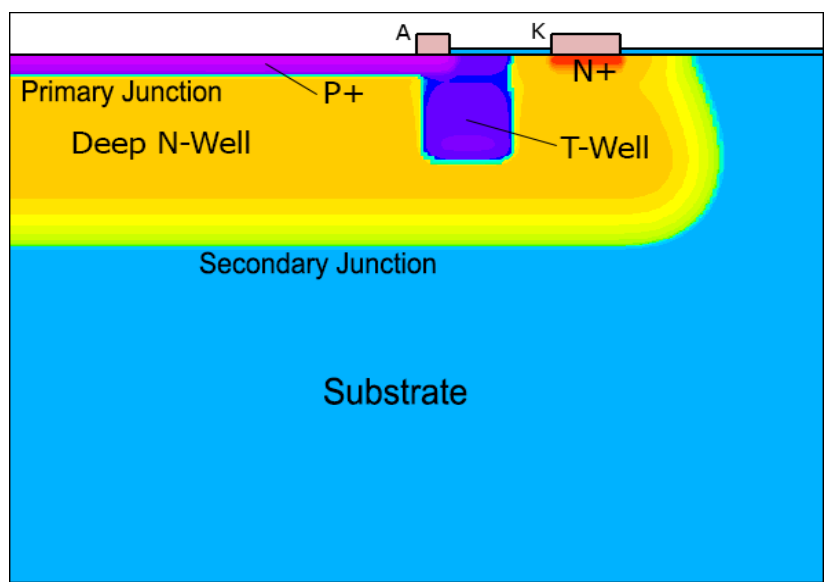

Fig. 2. SPAD structure simulated with TCAD tools. The left border acts as an axis of revolution [6].

Once the electron-hole pair have been created within the device, both carriers will undergo diffusion accelerating according to the electric field profile present in the SPAD, until they trigger the first impact ionization, which may lead to an avalanche, or recombine. The time it takes for an electric charge carrier to diffuse and provoke that first ionization is called diffusion time. After the first impact ionization, subsequent impact ionization occurs due to the accelerated charge carriers created in the first impact. In this point, the avalanche ignition may still fail. The time it takes to the avalanche to finally stabilize is called build-up time. Finally, after the avalanche is stabilized, it spreads throughout the active area of the SPAD. The time it takes for the avalanche to spread and reach a minimum current threshold detectable by the circuitry is the spread time. Altogether, they add to make the avalanche response time, whose statistical fluctuation is the photondetection timing jitter.

\section{A. Diffussion time}

Let us assume that this photon has been detected and have triggered an avalanche. Electron-hole pairs, able to originate an avalanche, can only be created in a determined zone of the SPAD as shown in Fig. 3. Here the module and direction of the electric field can be seen within a portion of active area region of the SPAD. Approximately 1-micrometer-deep, the vertical component of the electric field changes direction and carrier generated below that point are unable to reach the depletion region and trigger an avalanche, establishing in fact a sensing limit. On the other hand, any carrier created in any of the neutral regions may diffuse to the depletion region (electrons for the shallow region and holes for the deep region), accelerate until they reach the saturated drift velocity and trigger impact ionization which would result in an avalanche current. The time it takes for these carrier to reach the depletion zone and trigger the first impact ionization, that may or may not trigger an avalanche, is called the diffusion time. Notice that even carriers created in the depletion region, where electric field is maximum, have to diffuse and gain momentum to impactionize for the first time. Spinelli states that the minimum time for a carrier to gain this needed momentum is around 10 ps [8].

The first step to calculate this diffusion time is to make an estimation of the depth at which the photon has been absorbed. As we have assumed that the avalanche has taken place, the photon had to be absorbed no further than one-micrometer-deep in this case. To make a statistical law that generates a random depth to be absorbed, we define the current generated for incident photons of a given wavelength as [9]:

$$
\begin{gathered}
G=\eta_{0} \frac{P \lambda}{h c} \alpha e^{-\alpha y} \\
\alpha=\frac{4 \pi}{\lambda} k
\end{gathered}
$$

being $\alpha$ the absorption coefficient, $P$ is the ray intensity factor, which contains the cumulative effects of reflections, transmissions, and loss due to absorption over the ray path, $\eta_{0}$ is the internal quantum efficiency, which represents the number of carrier pairs generated per photon observed, $y$ the depth of absorption, and $k$ is the imaginary part of the optical index of refraction or extinction coefficient.

The main problem here is that the extinction coefficient, and therefore the absorption coefficient, is dependent on temperature and, more importantly, on the doping concentration. The extinction coefficient in low-doped silicon is well known and offer no significant variations when the doping concentration is less than $10^{17} \mathrm{~cm}^{-3}$. In this range the coefficient follows a quasi-

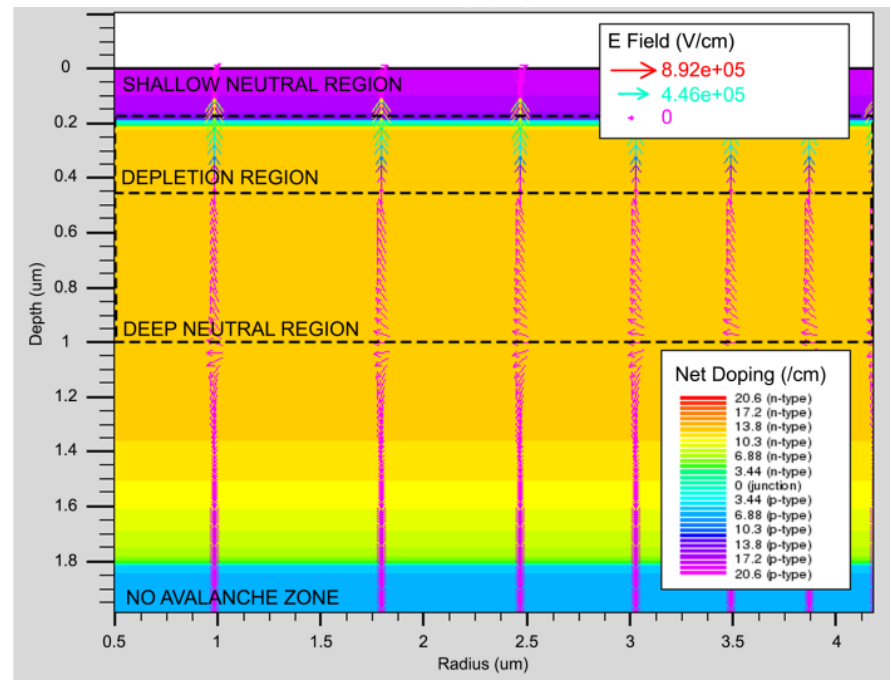

Fig. 3. Electric field profile in the SPAD active area region. 
exponential law in the optical spectrum [10]. However, our model contains regions where the doping concentration is far greater and therefore it has to be taken into account. Through TCAD simulation we have determined that the absorption coefficient in the active area region of our device can be adjusted to the following power law:

$$
\alpha(\lambda)=8.254 \times 10^{-26} \cdot \lambda^{-6.84}\left[\mathrm{~cm}^{-1}\right]
$$

where $\lambda$ is the wavelength. As we can see, Eq. (2) follows an exponential law. However, Verilog-A is not very versatile in handling the exponential distribution function. This was solved by drawing on a uniform distribution to easily generate an exponential distribution:

$$
\begin{aligned}
& P(\lambda)=\frac{-\ln (1-a \cdot U)}{\alpha(\lambda)} \\
& a=1-e^{-\alpha(\lambda) \cdot d}
\end{aligned}
$$

where $U$ is the uniform distribution, $a$ is a normalization value that establish the maximum probability of the uniform distribution, due to the fact that the photon absorption range does not cover all the space as we assumed that in was indeed absorbed in the $0-1 \mu \mathrm{m}$ range and therefore the probability must be limited, and $d$ is the absorption width of the sensitive area in centimeters, in this case $10^{-4} \mathrm{~cm}$. The inclusion of this last parameter makes the result of Eq. (5) directly the statistical fluctuation of the photon absorption depth.

Once the depth at which the electron-hole pair was created has been determined, the time needed for the charge carrier to diffuse to the depletion zone needs to be estimated as well. This can be done by extracting the carrier velocity profile from the TCAD model, and then integrating these velocity curves to arrive at the following expressions for the diffusion time for electrons in the shallow region and holes in the deep region, respectively:

$$
\begin{gathered}
t_{d n}=\frac{e^{-v_{n 2} \cdot Y}-e^{-v_{n 2} \cdot y_{0}}}{v_{n 1} \cdot v_{n 2}} \\
t_{d p}=\frac{\ln \left|v_{p 1} * Y+v_{p 2}\right|-\ln \left|v_{p 2}\right|}{v_{p 1}}
\end{gathered}
$$

being $Y$ the absorption depth, $y_{0}$ the shallow limit depth of the depletion region and $v_{n 1}, v_{n 2}, v_{p 1}$ and $v_{p 2}$, velocity parameters that depends linearly on the excess bias voltage. Fig. 4 shows the electron velocity profile against depth for SPAD active area region, which is similar for holes.

\section{B. Build-up time}

Since it is known that there is noise associated with the avalanche multiplication process [8] [11], a contribution to the timing jitter is expected to arise from this source. The current resulting from the first carrier, which is of $2 \cdot 10^{-8} \mathrm{~A}$, by the ongoing multiplication process is irregular and it is not stabilized until it reaches $10^{-6} \mathrm{~A}$, so the timing jitter of this contribution cannot be reduced by lowering the discriminator threshold, which is as low as $10^{-4} \mathrm{~A}$ in the literature. The time that takes

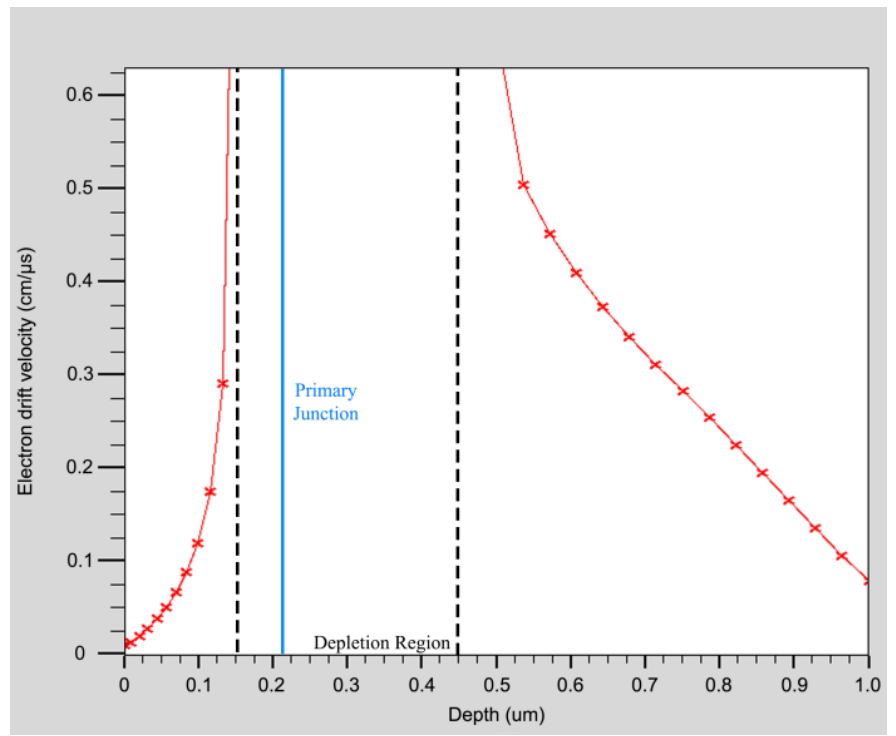

Fig. 4. Electron drift velocity (in red) vs Depth

the current to reach that level has a normal distribution and depends on the excess bias voltage and the electric field profile. As earlier works [8] [11], failed to match, experimental results of the build-up time at low excess bias voltages, we decided to define a piecewise relation with a power and a linear equation that fits Ingiargola's measurements [11], and is consistent with Spinelli's work [8]:

$$
t_{b, F W H M}=\left\{\begin{array}{c}
B_{1}(\varepsilon) \cdot V_{e}^{B_{2}(\varepsilon)}, V_{e}<V(\varepsilon) \\
B_{3}(\varepsilon) \cdot V_{e}+B_{4}(\varepsilon), V_{e} \geq V(\varepsilon)
\end{array}\right.
$$

being $\mathcal{E}$ the primary junction maximum electric field, $V_{e}$ the excess bias voltage, $B_{x}$ parameters that depend linearly on the electric field and $V(\mathcal{E})$ a potential value that represents the intersection point between the power and linear fitting, which has a quadratic dependence with the electric field.

Our results show that the primary electric field contribution dominates over the excess bias contribution to the build-up time. These results may lead to important decisions according to the device application, as engineering the primary junction to avoid an excessive high electric field junction and the subsequent noise due to band-to-band tunneling may lead to higher jitter due to the build-up time contribution.

\section{Spread time}

Once the avalanche is stable, it starts to spread laterally through the active area region. The model of the spreading velocity of the avalanche front for our SPAD is similar to that of [8], and is given by [12]:

$$
\begin{gathered}
v_{p}(r)= \begin{cases}v_{p 0}, & r<r_{0} \\
v_{p 0} r^{-k_{1}}, & r \geq r_{0}\end{cases} \\
v_{p 0}=2 \sqrt{\frac{D \cdot V_{E}}{\beta}}
\end{gathered}
$$

where $D$ is the diffusion coefficient of the charge carriers, $r$ is the avalanche front radius in micrometers, $r_{0} \approx 1 \mu \mathrm{m}$ four our 
devices, $V_{e}$ is the excess bias, $\beta$ is a constant that in our devices is $5.71 \times 10^{-12} \mathrm{ps} \mathrm{V}$, and $k_{1}=0.359$ is a constant that is related to spread of free carriers from the circular form of the avalanche spreading front.

Knowing the speed of the spreading of the avalanche front, allows us to calculate the percentage of the active area region of the SPAD that is undergoing avalanche at any given time, and thus the percentage of the avalanche maximum current.

\section{SIMULATION AND EXPERIMENTAL VALIDATION}

The starting point is the Verilog-A model of the SPAD based in TCAD simulations presented in [7]. A new version has been built to include all the previous considerations related to the timing jitter. Verilog-A simulations have been carried out using Cadence Spectre. The SPAD model was operated under a 100$\mathrm{k} \Omega$ passive quenching resistor.

During our research we found that the timing jitter of a SPAD was indeed heavily influenced by the threshold current of the quenching circuit as addressed by other works in literature [11][13]. If that threshold is low enough (below 2.5mA), then the timing jitter will be dominated by the build-up time, as the spread time would be negligible. Otherwise, the timing jitter is dominated by the latter. We have tried to find how our device behaves, supposed a minimum threshold current is imposed by the quenching circuitry. To do that we have limited the spreading area of the avalanche when the current has reached that threshold. Fig. 5 shows the timing jitter for various excessvoltages versus the anode voltage, $V_{A}$, that acts as an indicator of the threshold whichever the resistance on that terminal. The higher the threshold the higher the contribution of the spread time to the timing jitter. Also, a higher excess voltage improves the avalanche response, as both build-up and spreading develop faster. The results showed that under an excess bias of $0.9 \mathrm{~V}$, the $187 \mathrm{ps}$ of timing jitter obtained experimentally were also obtained for an anode voltage of $313 \mathrm{mV}$ corresponding to a threshold current of $6.26 \mathrm{~mA}$ with the avalanche covering the $52.5 \%$ of the SPAD active area. Interestingly, this implies that any avalanche whose maximum current, when it has expanded to the entire active area, is less than that of the threshold current of $6.26 \mathrm{~mA}$, will not be detected. As the maximum avalanche current is dependent on the excess bias, we can obtain a lower limit to the excess bias we have to apply to the detector so that it can detect an avalanche, being $470 \mathrm{mV}$ in this case. Indeed, if we take a look to the experimental photon detection efficiency (PDE) results in [6], it plummets close to $0.5 \mathrm{~V}$ excess bias, as the circuitry becomes unable to detect avalanches in the SPAD, which confirms the validity of our model. Fig. 5 also shows how the model behaves when an excess bias and electric field are forced to match under the same conditions Ingiargola's measurements [11]. In Fig. 6 an example of the timing jitter simulation can be seen. In this case the threshold current is $0.1 \mathrm{~mA}$ with a FWHM of $48 \mathrm{ps}$.

These results are compatible with other SPADs in literature. In [14], some devices of $8 \mu \mathrm{m}$ diameter are presented, all of them having greater timing jitters than the previous under and excess bias of $1.2 \mathrm{~V}$. The difference resides in the engineered primary junction of these SPADs which presents a lower electric field $(\sim 0.6 \mathrm{MV} / \mathrm{cm})$ than the one employed above $(0.729 \mathrm{MV} / \mathrm{cm})$.

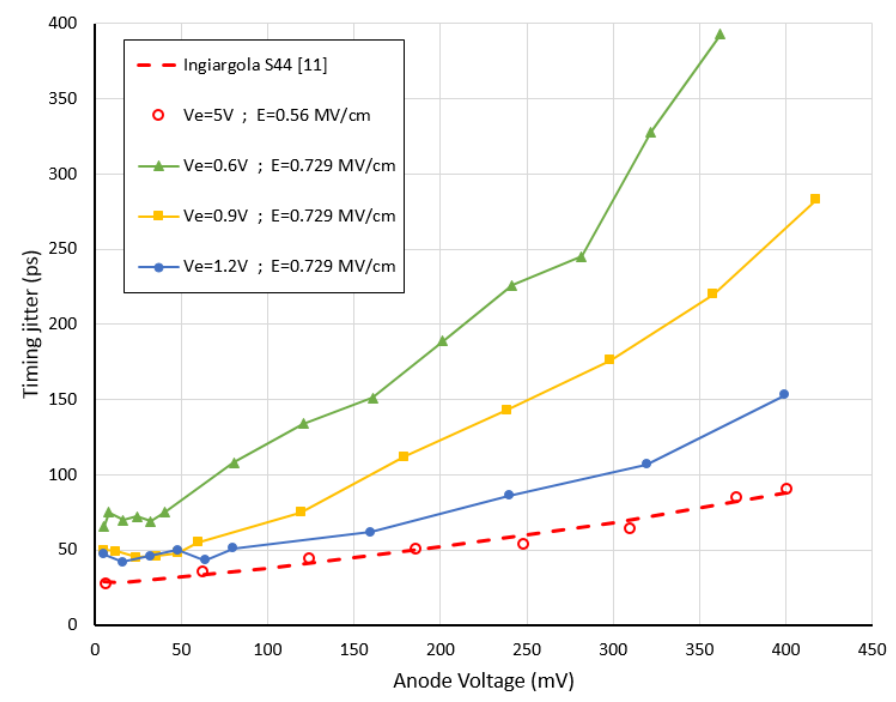

Fig. 5. Timing Jitter vs Anode Voltage for several excess bias voltages.

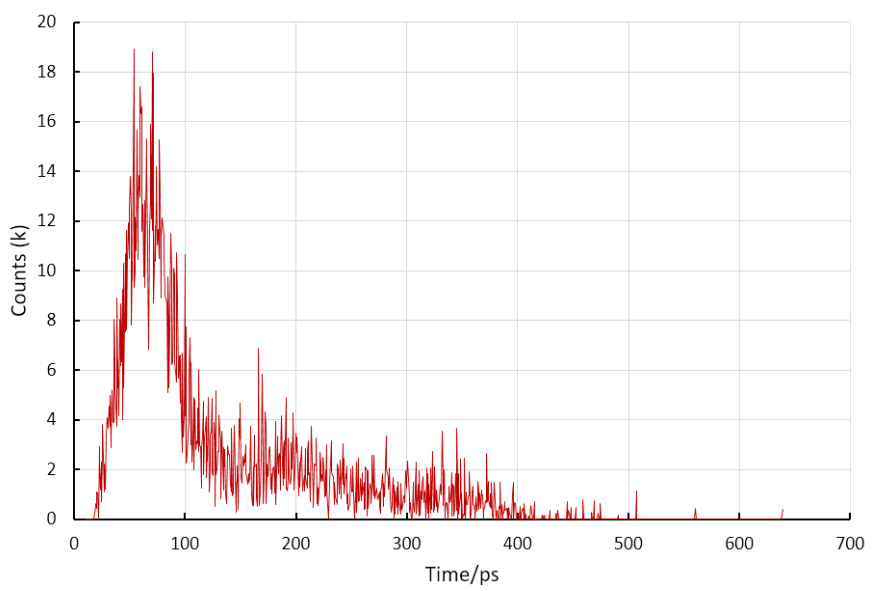

Fig. 6. Example of timing jitter results for a $20 \mathrm{~ms}$ run simulation, under $0.9 \mathrm{~V}$ of excess bias when the SPAD is illuminated with pulses of $80 \mathrm{ps}$ long, with a $447 \mathrm{~nm}$ wavelength beam with a frequency of $2.5 \mathrm{MHz}$.

\section{CONCLUSION}

The different mechanisms that contribute to the photondetection timing jitter have been explained. Results have been described as a consequence of including these mechanisms in a Verilog-A model. This model has proven to be an accurate tool to explain the possible reasons behind the timing jitter magnitude of some devices. It can also be employed to improve the circuits by lessening some of the contributions to photondetection timing jitter. For example, [13] suggested a method to detect the avalanche current long before it spreads throughout the SPAD, making the spread time contribution negligible. Also, the model can indicate if a system is suited for certain applications that need a low timing jitter.

\section{ACKNOWLEDGEMENT}

This work was funded by Junta de Andalucía's through grant TIC 2012-2338, the Spanish MINECO and European Region Development Fund (ERDF/FEDER) through Project RTI2018097088-B-C31, the US Office of Naval Research through Grant No. N00014-19-1-2156 and by EU H2020 MSCA through Project ACHIEVE-ITN (Grant No 765866). 


\section{REFERENCES}

[1] M. Bohmer and F. Pampaloni, "Time-resolved confocal scanning device for ultrasensitive fluorescence detection," Rev. Sci. Instr. vol. 11, no. 72, pp. 4145-4152, 2001.

[2] C. Niclass, C. Favi, T. Kluter, F.Monnier, and E. Charbon, "Singlesynchronous detection," IEEE J. Solid-State Circuits, vol. 44, no. 77, pp. 1977-1989, Sep. 2009.

[3] R. Mita, G. Pallumbo and P. G. Fallica, "Accurate model for singlephoton avalanche diodes," IET Circuits, Devices Syst., vol. 2, no. 2, pp. 207-212, Apr. 2008.

[4] G. Giustolisi, R. Mita and G. Pallumbo, "Behavioral modeling of statistical phenomena of single-photon avalanche diodes," Int. J. Circuit Theory Appl., vol. 40, no. 7, pp. 661-679, 2012.

[5] Z. Cheng, X. Cheng, D. Palubiak, M. J. Deen, and H. Peng, "A Comprehensive and Accurate Analytical SPAD Model for Circuit Simulation," IEEE Trans. Electron Devices, vol. 63, no. 5, pp. 19401948, May 2016.

[6] I. Vornicu, R. Carmona-Galán, B. Pérez-Verdú and Á. RodríguezVázquez, "Compact CMOS active quenching/recharge circuits for SPAD arrays," in Int. J. of Circuit Theory and Applicat., vol. 44, No. 4, pp. 917-928, Apr. 2015.

[7] J. M. López-Martínez, I. Vornicu, R. Carmona-Galán and A. RodríguezVázquez, "An Experimentally-Validated Verilog-A SPAD Model Extracted from TCAD Simulation,” 2018 25th IEEE ICECS, Jan 2019.
[8] A. Spinelli and A. L. Lacaita, "Physics and Numerical Simulation of Single Photon Avalanche Diodes." IEEE Trans. Electron Devices, vol. 44, no. 11, pp. 1931-1943, Nov 1997.

[9] Atlas User's Manual: Device Simulation Software. Silvaco Inc., Santa Clara, CA, 2016

[10] Aspnes, D.E. \& Studna, A.. (1983). Dielectric functions and optical parameters of $\mathrm{Si}, \mathrm{Ge}, \mathrm{GaP}, \mathrm{GaAs}, \mathrm{GaSb}, \mathrm{InP}, \mathrm{InAs}$, and $\mathrm{InSb}$ from 1.5 to 6.0 eV. Phys. Rev. B. 27. 10.1103/PhysRevB.27.985.

[11] A. Ingargiola et al. "Avalanche buildup and propagation effects on photon-timing jitter in Si-SPAD with non-uniform electric field", Proc. SPIE 7320, Advanced Photon Counting Techniques III, 73200K (29 April 2009).

[12] A. Lacaita, M. Mastrapasqua, and S. Vanoli, "Observation of avalanche propagation by multiplication assisted diffusion in p-n junctions," Appl. Phys. Lett., vol. 57, pp. 489-491, 1990.

[13] A. Gulinatti, P. Maccagnani, I. Rech, M. Ghioni, and S. Cova, "35 ps time resolution at room temperature with large area single photon avalanche diodes," Electron. Lett. 41, 272-274 (2005).

[14] J. A. Richardson, E. A. G. Webster, L. A. Grant, and R. K. Henderson, "Scaleable Single-Photon Avalanche Diode Structures in Nanometer CMOS Technology," IEEE Trans. Electron Devices, vol. 58, no. 7, pp. 2028-2035, Jul 2011. 